\title{
EJAM Editorial: 30th Anniversary Special Issue
}

Michael Ward has kindly invited us, as founders of EJAM to write a few words of introduction to this anniversary issue. At first, John Ockendon was Editor-in-Chief and Sam Howison was Assistant Editor but later we became equals. Throughout the founding of the journal, we received invaluable support and encouragement from David Tranah of Cambridge University Press. John's response to Michael's invitation was easy because he believes that the points he made in his message 'Thirteen years in the cockpit' (EJAM 2003, 14, pp. 1-2) still remain valid. This is despite the fact that the breadth of applicability of mathematics has increased by an order of magnitude and the increasingly prominent issue of the role and utility of mathematical modelling and analysis in the age of AI. The question of explainability in AI is every bit as mathematical as, say, the corresponding but much older questions posed by the numerical solution of problems for which little theory is yet available.

EJAM was launched at a time when the distinctive styles of different national mathematical traditions were clearly discernible. The intervening decades have seen a great deal of crossfertilisation, in which EJAM has played its part; the extent to which national styles remain is an interesting coffee time discussion point. Importantly in view of the journal's title, and despite political perturbations, the synergy between the mathematical styles of the UK and the EU is still a prominent driver of EJAM's intellectual trajectory. Equally, our contributors from around the world add to the mix and the Editorial Board has always had global coverage. No mathematics is excluded provided it meets the tests of quality, interest, novelty, applicability and relevance to the world around us.

Younger readers may have missed much of the techological revolution brought about by LaTeX and the Internet. In the early days of EJAM, all submissions were in hard copy and arrived by snail mail (often with interesting exotic postage stamps); we regularly received typewritten manuscripts with hand-drawn figures, and even one or two entirely hand-written papers. EJAM has (of course!) moved with the times, especially since John retired, and like all journals, it now faces new questions: not so much how to deal with the new technology as how to maintain a distinctive and valuable role in an age of freely available preprints. Above all, EJAM's future remains, as it always has been, crucially dependent on the scientific novelty and excitement of the papers it publishes, and for that we thank you, our authors, editors, referees and readers.

John Ockendon (Oxford), Sam Howison (Oxford)

\section{Anniversary Issue}

EJAM has traditionally been a leading journal for original work in areas of mathematics in which an understanding of the application requires the use of new and interesting mathematical ideas. One specific mandate of the journal over the past 30 years has been to highlight the interaction 
between mathematical methodologies and analysis, mathematical modelling and computational techniques for the qualitative and quantitative understanding of varous problems in continuum mechanics. However, over the years, the coverage of EJAM has evolved and broadened into new areas of applications, inspired by exciting real-world applications many of which have either a biological or probabilistic flavour.

One new central mission of EJAM since 2015 is to seek out and publish, on a periodic basis, special thematic issues on emerging topics that highlight new important frontiers of applied mathematics. Since 2015, EJAM has published special focus issues on 'Connections between Deep Learning and PDEs', 'Biofilms', 'Theory and Applications of Non-Smooth Dynamical Systems', 'Stochastic Analysis in Applications', 'Applied Optimal Transport', 'Mathematics of Crime and Security', 'Big Data and PDE', 'Network Analysis and Modelling' and 'Celebrating 75 Years'. The breadth of topics of these special issues showcase the ongoing relevance and evolution of mathematical methodologies and the need for the development of new mathematical tools and perspectives to treat an ever increasing range of problems.

This particular special issue of EJAM is a celebration of its 30th year of being a leading journal at the interface of mathematical modelling, analysis and computation. To recognise this occassion, this issue contains 10 invited articles that have been solicited from our current, past and inaugural editorial board members. Hopefully, this collection of articles, which contain a broad range of current topics in wave propagation, continuum mechanics, math biology and computation, will offer our readership a rich panorama of different perspectives in applied mathematics, and will pose open problems and new directions in hot topic areas. In closing, we offer our heartfelt gratitude to our authors, our editorial board members, our referees and of course to our readers, for their support of EJAM over the past 30 years. Undoubtedly, EJAM will continue to evolve in new directions, informed and inspired by new problems and challenges of our age.

The Co-Editors-in-Chief: Martin Burger (Friedrich-Alexander Universität ErlangenNürnberg), John King (Nottingham) and Michael Ward (UBC). 